\title{
SELECTION EFFECTS AND STRUCTURAL SYMMETRIES IN THE ORIENTATION BASED UNIFIED SCHEME
}

\author{
C. C. Onuchukwu ${ }^{1}$ and A. A. Ubachukwu ${ }^{2}$ \\ Received November 1 2018; accepted February 62019
}

\begin{abstract}
Using the projected linear size, $(D)$ as an orientation parameter, the armlength ratio, $(Q)$ and the lobe-luminosity ratio, $(R)$ as asymmetry parameters, we test the FR-II galaxies (Gs) and radio-loud quasar (Qs) unification scheme. Using the median values of our binned sample, our results, generally indicate that the $D$ of the radio sources are smaller at higher redshifts $z$ and at larger $Q$ values, with the $D$ of Qs smaller than those of Gs across all $Q, z$ and $R$ bins. Also, the $D$ of Gs appear smaller for lower values of $Q$, if $R \leq 1.0$ but become larger at higher values of $Q$ if $R>1.0$. For Qs, the $D$ decreases with increasing $Q, R$ and $z$. These results imply that the beaming effect is more important in Qs than in Gs. The $D-R$ regression analyses for different $Q$ and $z$ subsamples suggest the importance of factors other than beaming and orientation in the interpretation of the evolution of these radio sources.
\end{abstract}

\section{RESUMEN}

Con el tamaño lineal proyectado $(D)$ como parámetro para la orientación, el cociente de las longitudes de los brazos $(Q)$ y el cociente de las luminosides de los lóbulos $(R)$ como parámetros de asimetría, ponemos a prueba el esquema unificado para galaxias FR-II (GS) y cuasares radio-intensos (Qs). Usando las medianas para nuestras muestras agrupadas, encontramos que en general las $D$ de las radio fuentes son menores para corrimientos al rojo $z$ y valores $Q$ mayores, siendo las $D$ de los Qs menores que las de las Gs para todos los grupos de $Q, z$ y $R$. Las $D$ de las Gs son menores para valores menores de $Q$ si $R \leq 1.0$ pero aumentan para valores mayores de $Q$ si $R>1.0$. Para los Qs, las $D$ disminuyen al aumentar $Q, R$ y $z$. Estos resultados implican que el efecto de colimación es más importante para las Qs que para las Gs. Las regresiones $D-R$ para distintas submuestras de $Q$ y $z$ sugieren la importancia de otros efectos para interpretar la evolución de estos objetos, además de la colimación y la orientación.

Key Words: general: method — method: data analysis — miscellaneous: galaxies - galaxies: active

\section{INTRODUCTION}

Generally, Extragalactic Radio Sources (EGRS) are classified based on their observed geometric structures, and the amount and variability of the magnitude of radio power they emit (e.g. Fanarof \& Riley 1974; Scheuer \& Readhead, 1979; Barthel 1989). With improved observations and better theo-

\footnotetext{
${ }^{1}$ Department of Industrial Physics, Chukwuemeka Odumegwu Ojukwu University, Anambra State, Nigeria.

${ }^{2}$ Department of Physics and Astronomy, Faculty of Physical Sciences, Carver Building, University of Nigeria Nsukka, Enugu State, Nigeria.
}

retical foundation, the general understanding is that the nature and processes that govern the generation and dynamical evolution of EGRS is common to them all. Thus, unification schemes have been developed which posit that, fundamentally, all EGRS are similar in nature and differ only by the factors limiting/affecting their observations. These factors include the viewing angle, relativistic beaming, screens through which the sources are viewed; obscuring torus, time travel and time delay effects (Rees 1967; Ryle \& Longair 1967; Zensus 1997; Urry \& Padovani 1995; Laing 1988; Garrington et al. 1988; Garring- 
ton \& Conway 1991; Willot et al. 1998; Barthel 1989; Ubachukwu \& Ogwo, 1998; Antonucci 1993; Ubachukwu 2002; Gopal-Krishna \& Wiita 2004).

In the relativistic beaming and orientation based unification scheme, the projected linear size $D$ of a radio source is believed to be fore-shortened due to orientation effect and is given by (e.g. Ubachukwu 2002)

$$
D=D_{0} \sin \theta
$$

where $D_{0}$ in the intrinsic linear size of the radio source in its rest frame and $\theta$ the viewing angle with respect to a distant observer. In the relativistic beaming scenario, the observed arm-length ratio, $Q$, generally defined as the ratio of the approaching core-lobe length $D_{a}$ to that of the receding core-lobe length $D_{r}$, is given by (e.g. Rees 1967; Ryle \& Longair 1967; Ubachukwu 2002; Gopal-Krishna \& Wiita 2004)

$$
Q=\frac{D_{a}}{D_{r}}=\frac{1+\beta \cos \theta}{1-\beta \cos \theta},
$$

where $\beta$ is the bulk advance speed of the radio emitting plasma in unit of $c$ (the speed of light). Similarly, the observed lobe luminosity ratio $R$ is defined as the ratio of the luminosity of the lobe of the approaching arm to that of the receding arm, and is given by (e.g. Rees 1967; Ryle \& Longair 1967; Ubachukwu 2002; Gopal-Krishna \& Wiita 2004),

$$
R=\left(\frac{1+\beta \cos \theta}{1-\beta \cos \theta}\right)^{n+\alpha},
$$

where $n$ is a factor which depends on the jet flow model, where $n=3$ is assumed for jet consisting of blobs and $n=2$ is assumed for jets consisting of continuous flow. The spectral index, $\alpha$, is defined by $S_{(\nu)} \approx \nu^{-\alpha}$ where $S_{(\nu)}$ is the flux density at the frequency of observation $\nu$. From equations (1)-(3), it follows that as $\theta \longrightarrow \theta_{\min }, Q \longrightarrow Q_{\max }$ and $R \longrightarrow R_{\max }$ while $D \longrightarrow D_{\min }$; here $\min / \max$ is the minimum/maximum value (see Ubachukwu 2002; Onuchukwu \& Ubachukwu 2013). We therefore should expect some form of inverse $D-Q$ and $D-R$ relations from pure orientation arguments.

In the orientation based unification scheme of extragalactic radio sources, radio loud quasars and FR-II radio galaxies are expected to differ only in their orientation dependent properties, since FR-II radio galaxies are believed to lie closer to the plane of the sky than radio loud quasars (e.g. Barthel 1989). Thus, we expect a stronger anti-correlation between the projected linear size $(D)$ and the asymmetry parameters $(Q / R)$ for radio-loud quasars than for radio galaxies (Barthel 1989; Urry \& Padovani
$1995)$ if pure orientation is the only factor responsible for the differences between these two classes of radio sources. However, in flux density limited source samples, there is a strong selection effect due to the ubiquitous Malmquist bias which must be taken into account while considering quasars/galaxy unification schemes (e.g. Ubachukwu and Ogwo 1998).

In this paper, using the projected linear size as orientation parameter, the arm-length ratio and the lobe-luminosity ratio as asymmetry parameters, we test the FR-II radio galaxy and radio-loud quasar unification scheme for a well-defined sample (e.g Nilsson 1998), and the effect of selection bias in the two classes of radio sources in their luminosityredshift plane.

\section{DATA}

The data used for this analysis comprises 1045 heterogeneous sources (543 FR-II radio galaxies (Gs), 366 radio loud quasars (Qs) and 135 unidentified radio sources based on optical classification) taken from Nilsson (1998). For the present analysis, we selected the sources with complete information on the parameters: the redshift $(z)$, the log of luminosity at $178 \mathrm{MHz}\left(\log P_{178 \mathrm{MHz}}\right)$, the lobe-luminosity asymmetry parameter $(R)$, the arm-length asymmetry parameter $(Q)$, the spectral index $(\alpha)$ and the projected linear size of the radio source $(D)$. The final sample consists of 495 radio sources with 243 FR-II radio galaxies and 252 radio-loud quasars. We also form subsamples of highly asymmetric sources defined by $Q>1.5$ (93 radio quasars and 63 FR-II radio galaxies) and less asymmetric sources defined by $Q \leq 1.5$ (159 radio quasars and 180 FR-II radio galaxies). Though the break at $Q \leq 1.5 / Q>1.5$ was chosen arbitrarily, we are aware of the result obtained in Teerikorpi (2001), which suggests the existence of a correlation between radio source linear size and core luminosity for less asymmetric sources $(Q \leq 1.5)$ than for more asymmetric sources $(Q>1.5)$.

Figure 1 shows the $P-z$ plot for our sample which indicates a change in slope at about $z=0.3$. This change in slope believed to be due to selection effect is shown in Table 1. The quasar subsample with $z \leq 0.3$ indicates a negative $P-z$ slope while others are positive. In our sample, there are only 11 radio loud quasars with $z \leq 0.3$; this low number statistics may have been responsible for the observed negative slope. We note that several authors have pointed out the slope change in the $P / z$ relation from low $z$ radio sources to high $z$ radio sources (See Ubachukwu and Ogwo 1998; Onuchukwu 2014; 


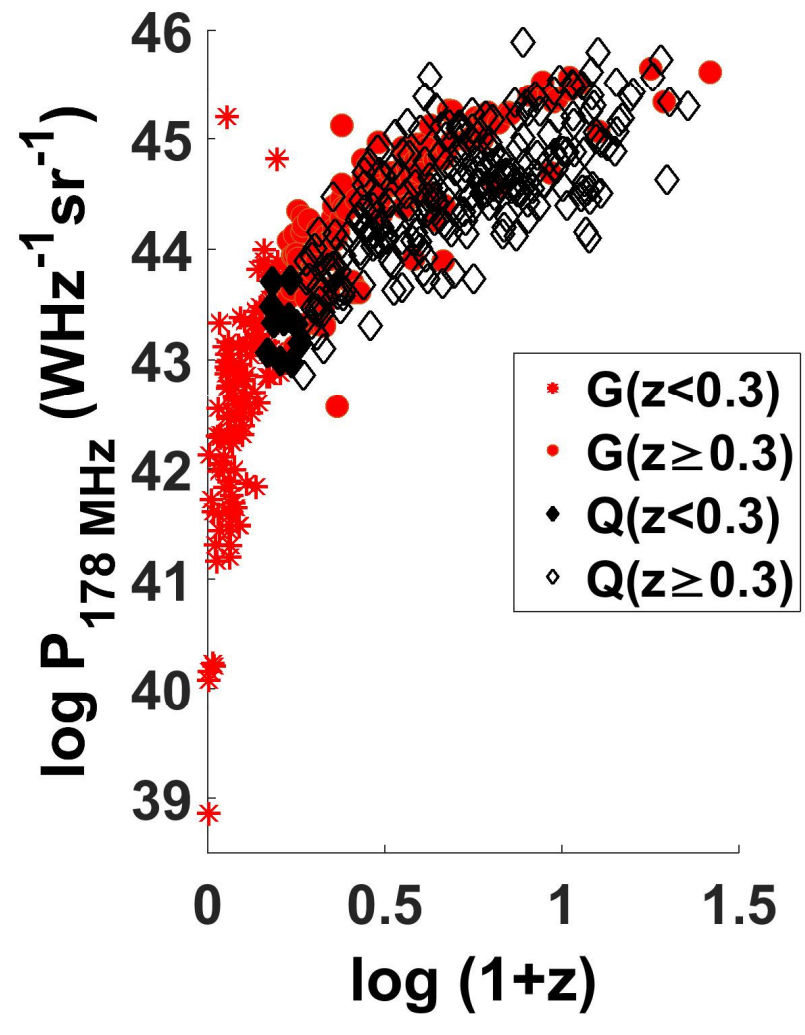

Fig. 1. Distribution plot $\log P_{178 \mathrm{MHz}}$ against $\log (1+z)$ for $z<0.3$ and $z \geq 0.3$ for galaxies (red) and quasars (black). The color figure can be viewed online.

Onah et al. 2018). Thus, we form a subsample of sources with $z \geq 0.3$ and compare the results of our analysis with those of the whole redshift range. There are 112 FR-II radio galaxies at $z \geq 0.3$ in our sample, with 77 having $Q \leq 1.5$ while 35 have $Q>1.5$. For the radio-loud quasars, we have 242 sources at $z \geq 0.3$, with 150 having $Q \leq 1.5$ while 92 have $Q>1.5$. For our analyses and results, we have the $z$-samples (for all $z$ and with $z \geq 0.3$ ) and two $Q$-subsamples (with $Q \leq 1.5$ and $>1.5$ ).

\section{ANALYSES, RESULTS AND DISCUSSION}

We show in Table 2 the results of the average values (the means with the associated error and the median values) for the analysed radio source parameters $(D, Q$ and $R)$ for the different ranges of $z$ and $Q$ subsamples. Generally, the projected linear sizes of galaxies $\left(D_{\mathrm{G}}\right)$ appear to be larger than those of quasars $\left(D_{\mathrm{Q}}\right)$. Based on the median value data, the ratio $D_{\mathrm{G}} / D_{\mathrm{Q}} \approx 1.7-1.8$ for the whole sample and for the two $Q$-subsamples ( for all $z$ ), but it decreases to $D_{\mathrm{G}} / D_{\mathrm{Q}} \approx 1.4-1.5$ for the $z$-subsample $(z \geq 0.3)$ and the two $Q$-subsamples (for $z \geq 0.3$ ). This is consistent with the quasar-galaxy unification scheme, in
TABLE 1

CORRELATION COEFFICIENT.*

\begin{tabular}{rrr}
\hline & \multicolumn{1}{c}{$r$} & \multicolumn{1}{c}{ Slope } \\
\hline $\mathrm{G}(z<0.3)$ & 0.7 & $26.2 \pm 0.7$ \\
$\mathrm{Q}(z<0.3)$ & -0.1 & $-2.6 \pm 0.3$ \\
$\mathrm{G}(z \geq 0.3)$ & 0.8 & $4.6 \pm 0.4$ \\
$\mathrm{Q}(z \geq 0.3)$ & 0.7 & $3.6 \pm 0.4$ \\
\hline
\end{tabular}

${ }^{*}$ Results and slope of the regression fit to $\log P_{178 \mathrm{MHz}}$ against $\log (1+z)$ for $z<0.3$ and $z \geq 0.3$.

which quasars are believed to be the beamed counterpart of radio galaxies which form the parent objects (e.g. Barthel 1989).

Generally, for the sample and $z$-subsamples considered, the ratio $Q_{\mathrm{G}} / Q_{\mathrm{Q}} \approx 1$ for various $Q$ subsamples. The similarity of $Q$-values for quasars and galaxies for different $Q$ and $z$ subsamples may be regarded as a pointer to the same micro-physics at work in the evolution of these radio sources (see Ryś 1994, 2000).

For all the samples and $z$-subsamples considered, and for the $Q \leq 1.5$ subsample, the ratio $R_{\mathrm{G}} / R_{\mathrm{Q}} \approx 1$ but it decreases to $R_{\mathrm{G}} / R_{\mathrm{Q}} \approx 0.6$ for the $Q>1.5$ subsample. The observed similarities/differences between the lobe-luminosity ratio of galaxies and quasars for $Q \leq 1.5 / Q>1.5$ suggest that the beaming/orientation effects that foreshorten the observed projected size of quasars (note $D_{\mathrm{G}} / D_{\mathrm{Q}}$ is systematically smaller for $Q>1.5$ than $Q \leq 1.5$ - see the median values) may also be responsible for enhancing its brightness, thus the lower values of $R_{\mathrm{G}} / R_{\mathrm{Q}}$ obtained for $Q>1.5$. We also note that several authors (e.g. Ingham \& Morrison 1975; Valtonen 1979; Macklin 1981; McCarthy et al. 1991; Best et al. 1995; Wardle \& Aaron 1997; Jeyakumar \& Saikia 2000; Saikia et al. 2003; Arshakian \& Longair 2004; Jeyakumar et al. 2005; Subrahmanyan et al. 2008; Safouris et al. 2009; Priya et al. 2012; Onuchukwu \& Ubachukwu 2013; Onuchukwu 2017)) have pointed at other factors (e.g. environment/intrinsic) as being important in the interpretation of the dynamics and evolution of such highly asymmetric radio sources. We also observe that the quasars in our sample are located in denser environments than galaxies (the density $(\rho)$ of the universe scales as $\rho \propto(1+z)^{x}$, where $x$ is positive), with the median redshift for quasars and galaxies in our sample being 1.1 and 0.3 respectively; while for the subsample with $z \geq 0.3$, the median redshift for the galaxy subclass is $\approx 0.8$. and that of the quasar subclass is $\approx 1.1$. 
TABLE 2

AVERAGE VALUES (MEAN WITH ASSOCIATED ERROR \& MEDIAN) OF THE PROJECTED LINEAR SIZE $(D){ }^{*}$

\begin{tabular}{|c|c|c|c|c|c|c|c|}
\hline$z$ Range & & $\begin{array}{c}D(\mathrm{kpc}) \\
\text { mean }\end{array}$ & $\begin{array}{l}D(\mathrm{kpc}) \\
\text { median }\end{array}$ & $\begin{array}{c}Q \\
\text { mean }\end{array}$ & $\begin{array}{c}Q \\
\text { median }\end{array}$ & $\begin{array}{c}R \\
\text { mean }\end{array}$ & $\begin{array}{c}R \\
\text { median }\end{array}$ \\
\hline$z(\mathrm{All})$ & Gs $(\operatorname{All} Q)$ & $401.5 \pm 308.4$ & 270.0 & $1.4 \pm 0.3$ & 1.3 & $1.1 \pm 0.6$ & 0.9 \\
\hline$z(\mathrm{All})$ & Qs $($ All $Q)$ & $221.4 \pm 162.0$ & 146.6 & $1.6 \pm 0.5$ & 1.3 & $2.0 \pm 1.8$ & 1.0 \\
\hline$z(\mathrm{All})$ & $\operatorname{Gs}(Q \leq 1.5)$ & $380.2 \pm 255.8$ & 300.3 & $1.2 \pm 0.1$ & 1.2 & $1.1 \pm 0.5$ & 0.9 \\
\hline$z(\mathrm{All})$ & $\operatorname{Qs}(Q \leq 1.5)$ & $257.8 \pm 181.8$ & 167.0 & $1.2 \pm 0.1$ & 1.2 & $2.2 \pm 2.1$ & 1.0 \\
\hline$z(\mathrm{All})$ & $\operatorname{Gs}(Q>1.5)$ & $462.2 \pm 465.5$ & 185.0 & $2.0 \pm 0.4$ & 1.8 & $1.1 \pm 0.8$ & 0.7 \\
\hline$z($ All $)$ & $\operatorname{Qs}(Q>1.5)$ & $159.3 \pm 115.4$ & 106.3 & $2.2 \pm 0.7$ & 1.8 & $1.6 \pm 1.3$ & 1.2 \\
\hline$z \geq 0.3$ & Gs $($ All $Q)$ & $310.6 \pm 222.3$ & 223.9 & $1.5 \pm 0.4$ & 1.3 & $1.2 \pm 0.8$ & 0.9 \\
\hline$z \geq 0.3$ & Qs $($ All $Q)$ & $205.9 \pm 146.8$ & 141.5 & $1.6 \pm 0.5$ & 1.3 & $2.0 \pm 1.8$ & 1.0 \\
\hline$z \geq 0.3$ & $\operatorname{Gs}(Q \leq 1.5)$ & $322.6 \pm 213.7$ & 241.0 & $1.2 \pm 0.1$ & 1.2 & $1.2 \pm 0.6$ & 1.0 \\
\hline$z \geq 0.3$ & $\operatorname{Qs}(Q \leq 1.5)$ & $234.8 \pm 160.4$ & 160.1 & $1.2 \pm 0.1$ & 1.2 & $2.2 \pm 2.2$ & 1.0 \\
\hline$z \geq 0.3$ & $\operatorname{Gs}(Q>1.5)$ & $284.4 \pm 236.9$ & 151.9 & $2.2 \pm 0.5$ & 2.0 & $1.4 \pm 1.2$ & 0.7 \\
\hline$z \geq 0.3$ & $\operatorname{Qs}(Q>1.5)$ & $158.8 \pm 116.0$ & 105.5 & $2.2 \pm 0.7$ & 1.8 & $1.7 \pm 1.3$ & 1.2 \\
\hline
\end{tabular}

* Redshift and $\log P_{178 \mathrm{MHz}}$ estimated using all the sources in the sample for radio-loud quasars and FR-II radio galaxies, and for the sample and the subsample with $z \geq 0.3$.

TABLE 3

CORRELATION COEFFICIENT RESULTS FOR $D-Q / R{ }^{*}$

\begin{tabular}{|c|c|c|c|c|c|}
\hline & & Gs & Qs & Gs & Qs \\
\hline & $R$ (All) & $D / Q$ & $D / Q$ & $D / R$ & $D / R$ \\
\hline$z$ (All) & $Q(\mathrm{All})$ & -0.2 & -0.2 & 0.1 & -0.1 \\
\hline$z($ All $)$ & $Q \leq 1.5$ & 0.0 & 0.0 & 0.1 & -0.2 \\
\hline$z$ (All) & $Q>1.5$ & -0.2 & -0.1 & 0.1 & 0.0 \\
\hline$z \geq 0.3$ & $Q(\mathrm{All})$ & -0.3 & -0.2 & 0.2 & -0.1 \\
\hline$z \geq 0.3$ & $Q \leq 1.5$ & -0.2 & 0.0 & 0.2 & -0.2 \\
\hline \multirow[t]{2}{*}{$z \geq 0.3$} & $Q>1.5$ & -0.4 & -0.1 & 0.3 & 0.1 \\
\hline & $R>1.0$ & & & & \\
\hline$z(\mathrm{All})$ & $Q(\mathrm{All})$ & -0.2 & -0.2 & 0.1 & -0.3 \\
\hline$z(\mathrm{All})$ & $Q \leq 1.5$ & -0.2 & -0.1 & 0.1 & -0.5 \\
\hline$z(\mathrm{All})$ & $Q>1.5$ & -0.3 & 0.0 & 0.2 & 0.1 \\
\hline$z \geq 0.3$ & $Q(\mathrm{All})$ & -0.4 & -0.2 & 0.0 & -0.3 \\
\hline$z \geq 0.3$ & $Q \leq 1.5$ & -0.5 & -0.1 & -0.2 & -0.5 \\
\hline \multirow[t]{2}{*}{$z \geq 0.3$} & $Q>1.5$ & -0.1 & 0.0 & 0.5 & 0.1 \\
\hline & $R \leq 1.0$ & & & & \\
\hline$z($ All $)$ & $Q(\mathrm{All})$ & -0.1 & -0.3 & 0.2 & 0.2 \\
\hline$z(\mathrm{All})$ & $Q \leq 1.5$ & 0.1 & 0.1 & 0.2 & 0.1 \\
\hline$z($ All $)$ & $Q>1.5$ & -0.2 & -0.2 & 0.1 & 0.1 \\
\hline$z \geq 0.3$ & $Q(\mathrm{All})$ & -0.2 & -0.3 & 0.2 & 0.2 \\
\hline$z \geq 0.3$ & $Q \leq 1.5$ & 0.1 & 0.1 & 0.1 & 0.1 \\
\hline$z \geq 0.3$ & $Q>1.5$ & -0.5 & -0.2 & 0.4 & 0.1 \\
\hline
\end{tabular}

*Relations for different redshift and $Q$ subsamples for radio-loud quasars and FR-II radio galaxies.
In Figures 2-7 we display the distribution plots of $D, Q$ and $R$ of the radio sources for different $Q$ and $z$ subsamples. In Figure 2, the distribution plots of $D$ for all $Q, Q \leq 1.5$ and $Q>1.5$ for the sample and the $z$-subsample indicate a lognormal distribution for both galaxies and quasars, though with a seemingly increasing tendency to be more left-skewed for the galaxy subclass than for the quasar subclass of radio sources. We have used the natural logarithm considering the range in values of $D$ in our sample 1.1 $5853.3 \mathrm{kpc}$.

At $z>0.3$, there is a noticeable difference in the distribution of the linear sizes of galaxies and quasars (shown in Figure 3), which seems to increase with increasing $Q$. For quasars at all $Q(Q \leq 1.5$ and $Q>1.5$ combined) sample, the $D$ distribution approximates lognormal; but for the galaxies, for $Q>1.5$ subsample, the projected linear size distribution seems constant from above $100 \mathrm{kpc}$ to more than $1000 \mathrm{kpc}$. The remarkable differences in the distribution of $D$ for $Q>1.5 ; z>0.3$ between galaxies and quasars may be due to selection and beaming effects. At large $z$, it is expected that only relatively large sized galaxies of higher luminosity will be easily observable (the median luminosity of galaxies for $Q>1.5, z \geq 0.3$ is a factor of 7 higher than that of $Q>1.5, z<0.3)$. The median redshift for $Q>1.5 ; z>0.3$ is: for quasars $\left(z_{\text {median }} \approx 1.0\right)$ and for galaxies $\left(z_{\text {median }} \approx 0.7\right)$. Environmental differ- 

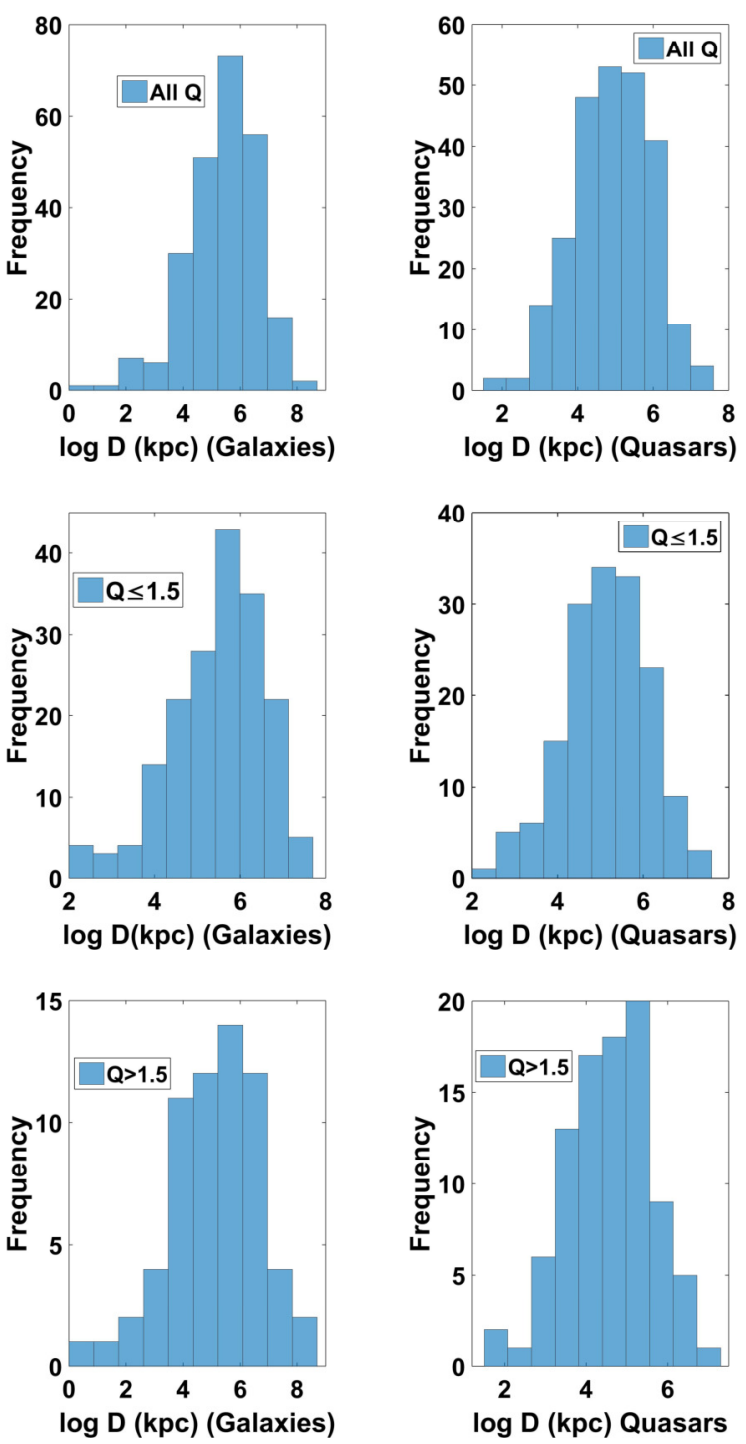

Fig. 2. Distribution plots of $\log D$ for galaxies and quasars for all $Q$ (Top Panel), $Q \leq 1.5$ (Centre Panel), and $Q>1.5$ (Bottom Panel) for all $z$.

ences, if present in the host galaxies, may be responsible for this observed difference, since the density of the universe scales as $\rho \approx(1+z)^{x}$. By implication, the observed quasars in our sample are found in more dense environments and may suffer greater restraint in expanding to all possible dimensions, while galaxies suffer less restraint and may attain all possible sizes.

Figure 4 shows the distribution plots of $Q$ for the whole sample and the $z$-subsample.The distributions appear similar for quasars and galaxies for all the different $Q$-subsamples (right skewed for $Q>1.5$ and for all $Q$ subsamples but seem to have a constant
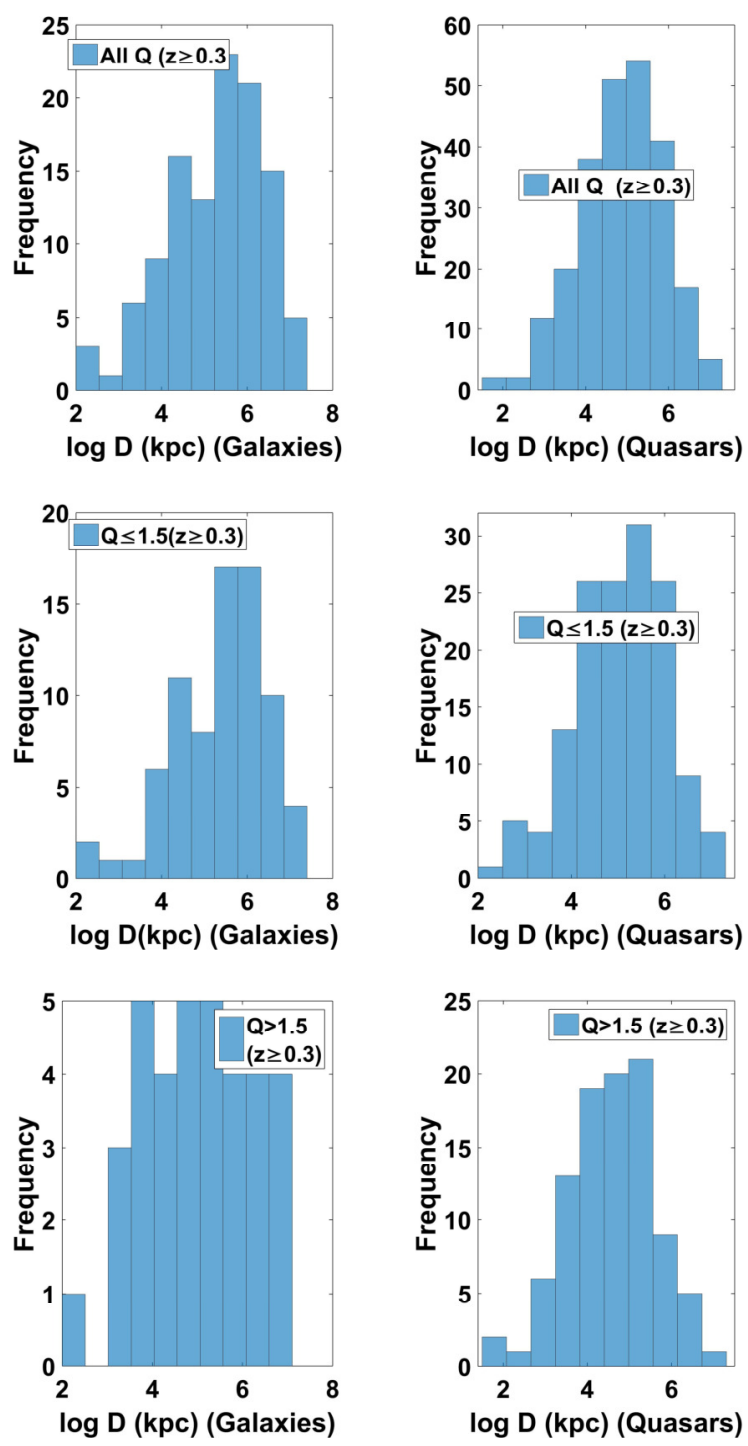

Fig. 3. Distribution plots of $\log D$ for galaxies and quasars for all $Q$ (Top Panel), $Q \leq 1.5$ (Centre Panel), and $Q>1.5$ (Bottom Panel) for the $z \geq 0.3$ subsample.

distribution for the subsample $1 \leq Q \leq 1.5$ ). For the $z>0.3$ subsample, (see Figure 5), the $Q$ distributions for the quasar and galaxy subclasses are similar as is the case for the all $-z$ sample. In Figures 6 and 7 we display the distribution plots of lobeluminosity ratio, $R$, for radio-loud quasars and FRII radio galaxies for the whole samples and various $z$ and $Q$ subsamples. The distributions appear lognormal for both classes of object. These distributions show that the observed asymmetries must have been caused by a number of independent factors (relativistic beaming, selection effects, environmental effects, etc.). 

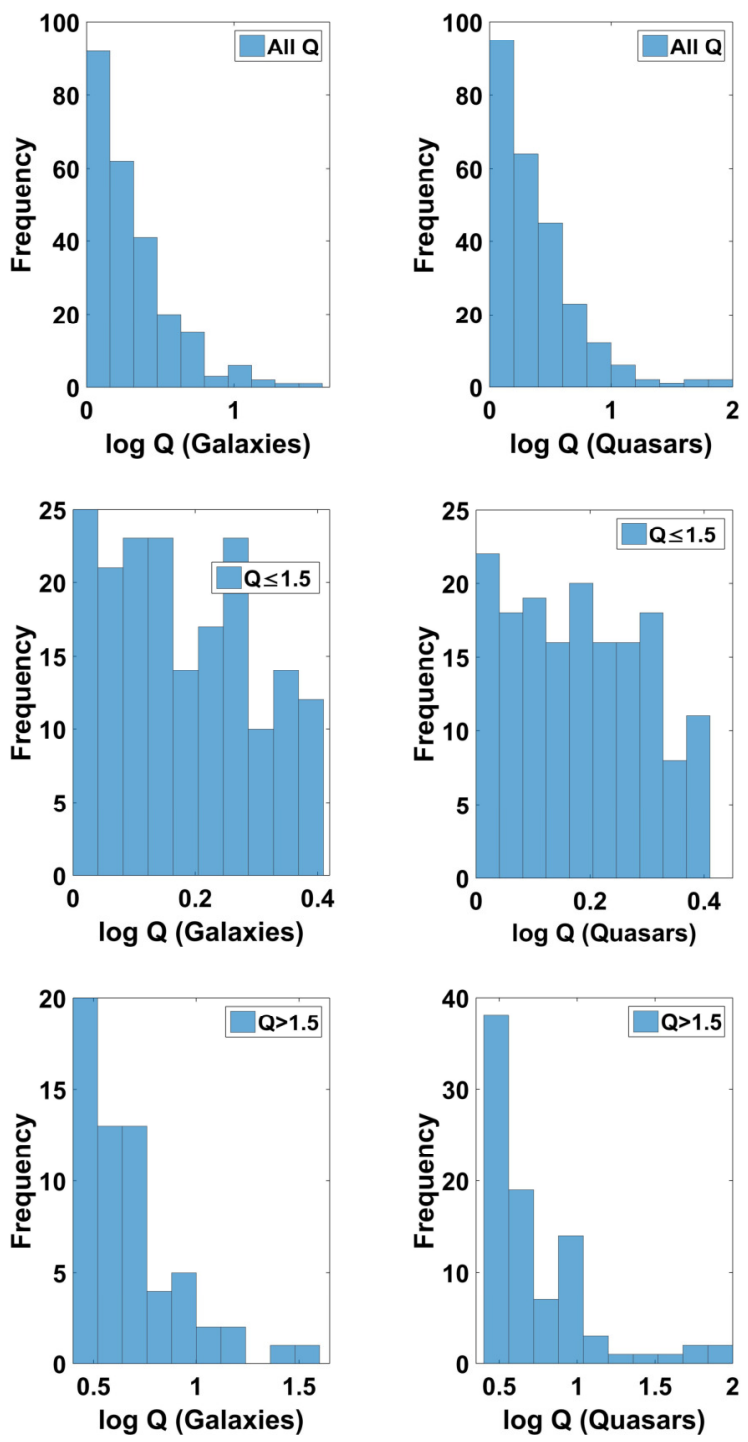

Fig. 4. Distribution plots of $Q$ for galaxies and quasars for all $Q$ (Top Panel), $Q \leq 1.5$ (Centre Panel), and $Q>1.5$ (Bottom Panel) for all $z$.

In Figures 8 and 9 we show the $D-Q / R$ data for the different $z$ and $Q$ subsamples. The results of the one-dimensional regression analyses of the plots are shown in Table 3. Equations (2) and (3) imply that if $Q \geq 1$, then $R \geq 1$. Our sample however shows that $49 \%$ of the quasars and $57 \%$ of the galaxies have $R \leq 1$, implying that relativistic beaming alone cannot explain the observed $R$-data. We have further subdivided our sample and subsamples to include sources with $R \leq 1.0$ and $R>1.0$ (see Table 3 ). We hereby show the average values of the observed parameters in Table 4, the orientation and the asymmetry parameters in Table 5 (in form of the ratios of
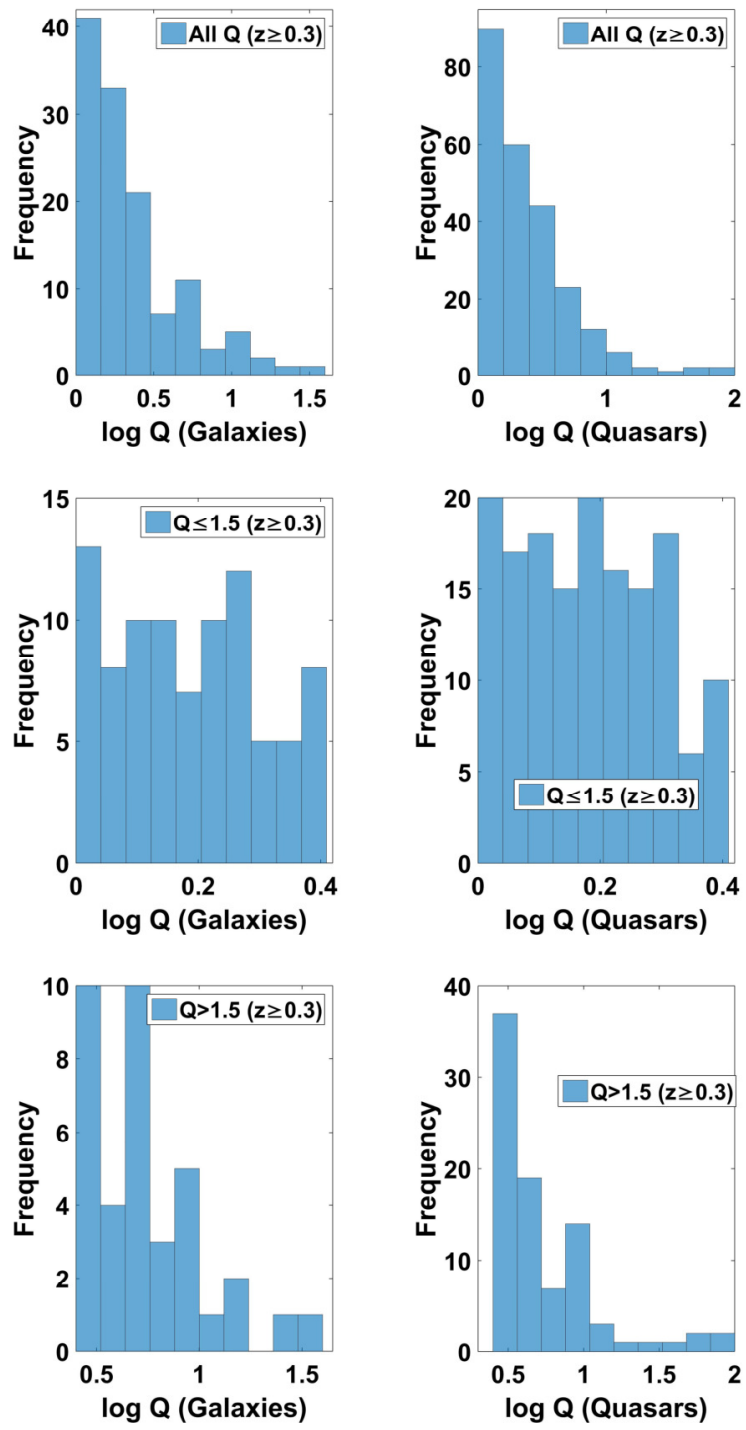

Fig. 5. Distribution plots of $Q$ for galaxies and quasars for all $Q$ (Top Panel), $Q \leq 1.5$ (Centre Panel), and $Q>1.5$ (Bottom Panel) for the $z \geq 0.3$ subsample.

$D_{\mathrm{G}} / D_{\mathrm{Q}}, Q_{\mathrm{G}} / Q_{\mathrm{Q}}$ and $R_{\mathrm{G}} / R_{\mathrm{Q}}$ for the different $z$ and $Q$ subsamples) and the matrix of their correlations in Table 6.

Analyses based on $R>1.0$ follow the same trend as that of the sample where all the $R$ values were used but with a slight improvement in the strength of the correlation, especially for the quasar subsample with $Q \leq 1.5$. For the subsample based on $R \leq 1.0$, both galaxy and quasar subclasses showed no $D-R$ correlation, and a slight inverse $Q-D$ correlation for the galaxy subclass for $z \geq 0.3 ; Q>1.5$. Actually, Onuchukwu (2017) pointed out that environmental/intrinsic factors (e.g. dense environment) may 

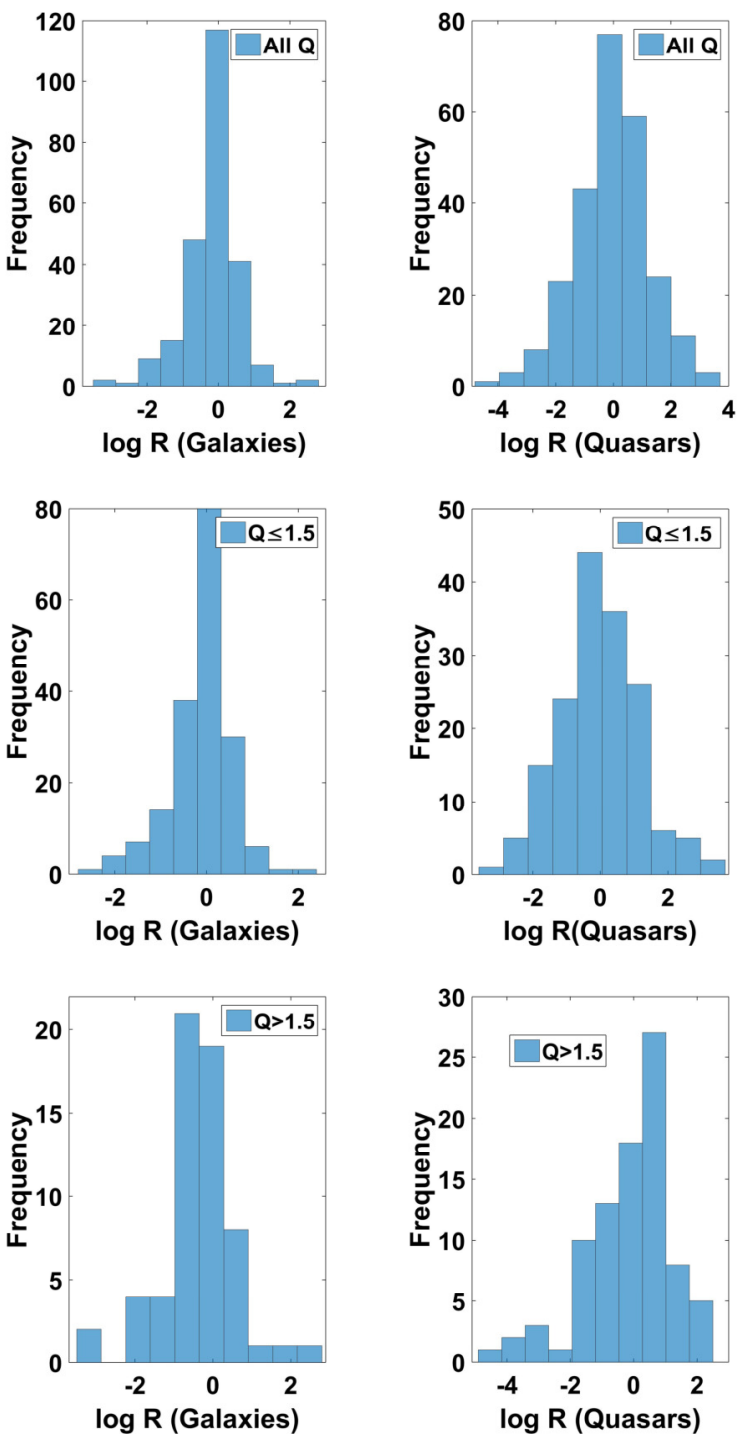

Fig. 6. Distribution plots of $R$ for galaxies and quasars for all $Q$ (Top Panel), $Q \leq 1.5$ (Centre Panel), and $Q>1.5$ (Bottom Panel) for all $z$.

limit the growth of one arm, thereby impeding large values of $Q$ and $D$, and will also enhance the luminosity of the arm (thereby increasing/decreasing the value of $R$ depending on whether it is assumed to be the approaching/receding side). In Nilsson (1998), the longer side, which may not be the physically approaching side, is assumed to be the approaching side (there was no determination of the jet/counterjet in the sources of the sample).

Table 4 indicates that linear sizes of FR-II radio galaxies depend on both $Q$ and $R$; being larger for less asymmetric sources $(Q \leq 1.5)$ than for more asymmetric sources $(Q>1.5)$ with $R \leq 1.0$ and
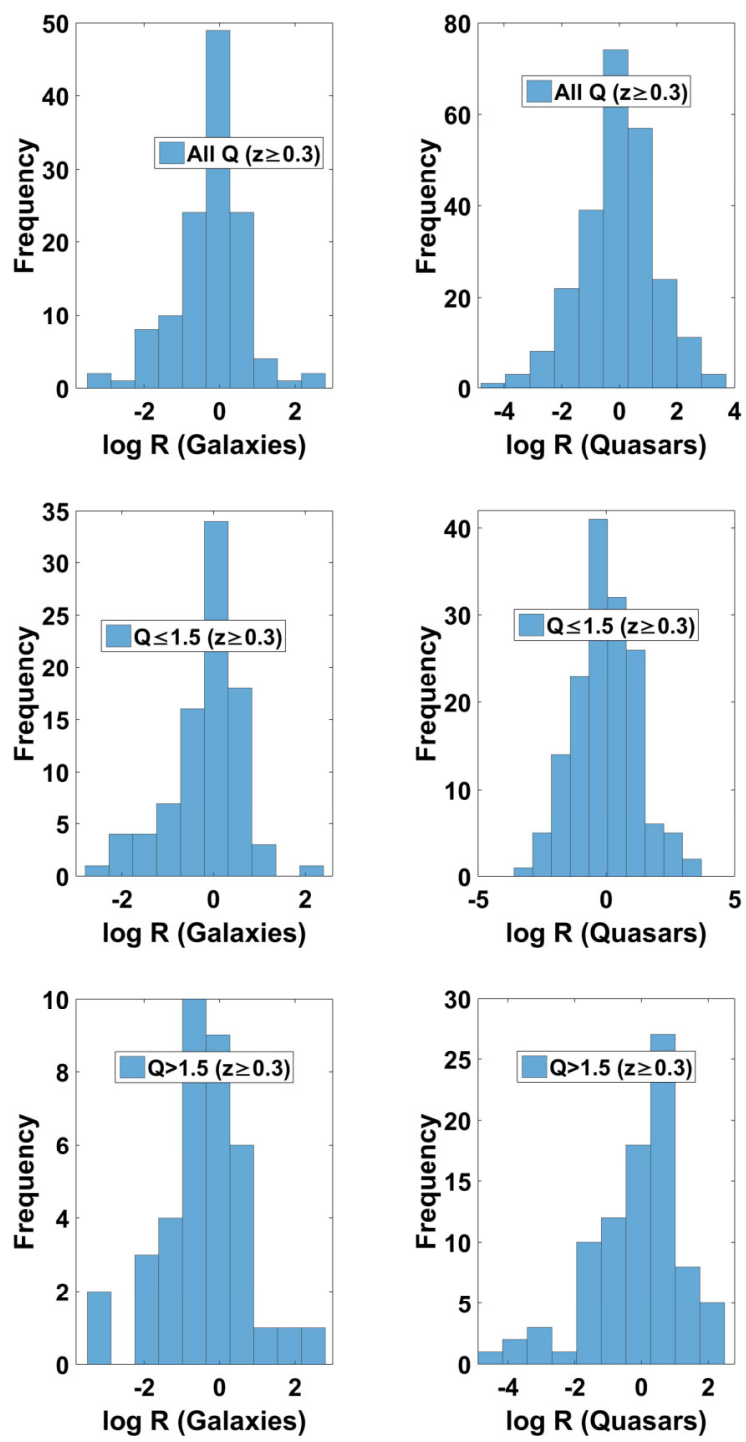

Fig. 7. Distribution plots of $R$ for galaxies and quasars for all $Q$ (Top Panel), $Q \leq 1.5$ (Centre Panel), and $Q>1.5$ (Bottom Panel) for the $z \geq 0.3$ subsample.

the reverse for $R>1.0$. For the quasar subclass, $D$ decreases with $Q$ independent of $R$. From Table 5, it can be seen that the asymmetry ratio $Q_{\mathrm{G}} / Q_{\mathrm{Q}}$ appears independent of $R$ and $z$, while $R_{\mathrm{G}} / R_{\mathrm{Q}}$ appears dependent on $z$. If radio loud quasars and FR-II radio galaxies differ by simple orientation, these ratios are expected to be independent of $z$, especially for all sources with $R>1.0$. Similarly, the orientation parameter $D_{\mathrm{G}} / D_{\mathrm{Q}}$ is expected to be dependent on $Q$ and $R$ for all $R>1.0$ but independent of $z$ for all sources with $z \geq 0.3$. This is generally true from Table 5 , where it can be seen that $D_{\mathrm{G}} / D_{\mathrm{Q}}$ is smaller for more asymmetric than for less asymmetric sources. 

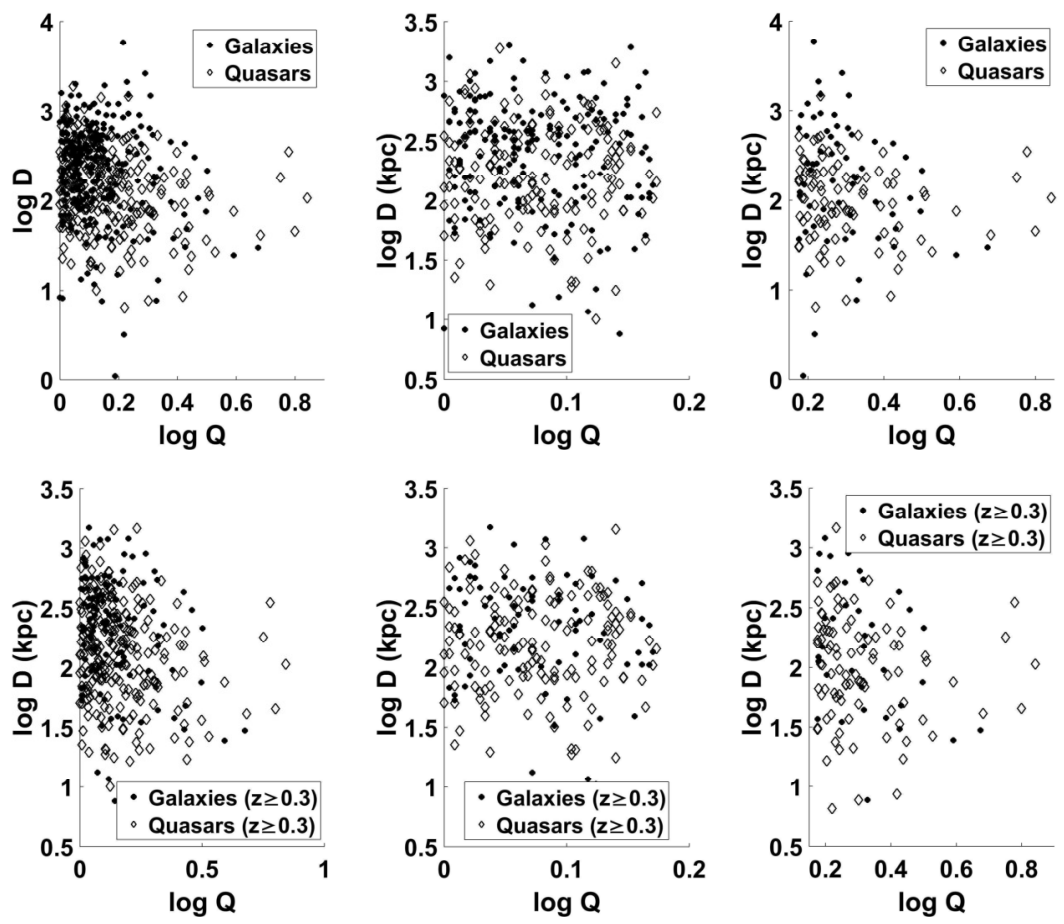

Fig. 8. $\log -\log$ plots of $D$ against $Q$ for galaxies and quasars for all $Q$ (Right), $Q \leq 1.5$ (Centre), and $Q>1.5$ (Left) for all $z$ (Top Panel) and for the $z \geq 0.3$ subsample (Bottom Panel).
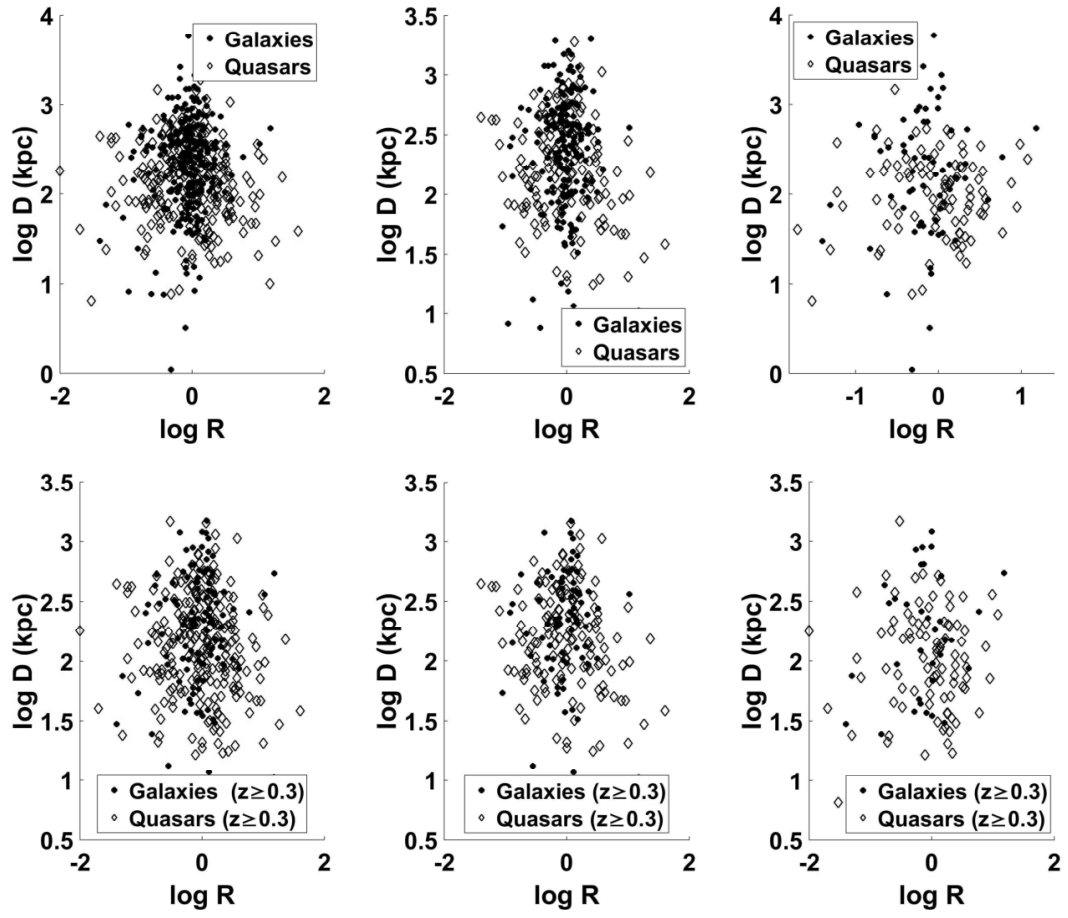

Fig. 9. $\log -\log$ plots of $D$ against $R$ for galaxies and quasars for all $Q$ (Right), $Q \leq 1.5$ (Centre), and $Q>1.5$ (Left) for all $z$ (Top Panel) and for the $z \geq 0.3$ subsample (Bottom Panel). 
TABLE 4

AVERAGE VALUES OF $D, Q$, AND $R .^{*}$

\begin{tabular}{|c|c|c|c|c|c|c|c|}
\hline & & $D$ & $D$ & $Q$ & $Q$ & $R$ & $R$ \\
\hline Galaxy & Mean & $R \leq 1.0$ & $R>1.0$ & $R \leq 1.0$ & $R>1.0$ & $R \leq 1.0$ & $R>1.0$ \\
\hline$z$ (all) & QAll & 399.5 & 398.7 & 1.5 & 1.3 & 0.6 & 1.8 \\
\hline$z($ all $)$ & $Q \leq 1.5$ & 359.8 & 406.6 & 1.2 & 1.2 & 0.7 & 1.7 \\
\hline$z$ (all) & $Q>1.5$ & 488.1 & 364.9 & 2.1 & 1.9 & 0.5 & 2.5 \\
\hline$z \geq 0.3$ & QAll & 268.6 & 346.0 & 1.6 & 1.4 & 0.5 & 2.1 \\
\hline$z \geq 0.3$ & $Q \leq 1.5$ & 251.1 & 400.0 & 1.2 & 1.2 & 0.6 & 1.8 \\
\hline$z \geq 0.3$ & $Q>1.5$ & 300.3 & 188.3 & 2.3 & 2.0 & 0.5 & 3.0 \\
\hline Quasar & Mean & & & & & & \\
\hline$z$ (all) & QAll & 229.3 & 216.8 & 1.6 & 1.6 & 0.5 & 3.5 \\
\hline$z$ (all) & $Q \leq 1.5$ & 251.6 & 270.6 & 1.2 & 1.2 & 0.5 & 4.0 \\
\hline$z$ (all) & $Q>1.5$ & 187.3 & 135.2 & 2.2 & 2.2 & 0.4 & 2.7 \\
\hline$z \geq 0.3$ & QAll & 221.2 & 194.1 & 1.6 & 1.6 & 0.5 & 3.5 \\
\hline$z \geq 0.3$ & $Q \leq 1.5$ & 240.2 & 235.0 & 1.2 & 1.2 & 0.5 & 4.1 \\
\hline$z \geq 0.3$ & $Q>1.5$ & 186.8 & 135.2 & 2.2 & 2.2 & 0.4 & 2.7 \\
\hline Galaxy & Median & & & & & & \\
\hline$z$ (all) & QAll & 255.0 & 276.0 & 1.3 & 1.2 & 0.7 & 1.3 \\
\hline$z$ (all) & $Q \leq 1.5$ & 282.4 & 312.0 & 1.2 & 1.1 & 0.7 & 1.3 \\
\hline$z($ all $)$ & $Q>1.5$ & 228.6 & 151.9 & 1.9 & 1.8 & 0.6 & 1.4 \\
\hline$z \geq 0.3$ & QAll & 189.7 & 256.4 & 1.3 & 1.2 & 0.6 & 1.4 \\
\hline$z \geq 0.3$ & $Q \leq 1.5$ & 178.5 & 327.0 & 1.2 & 1.2 & 0.6 & 1.4 \\
\hline$z \geq 0.3$ & $Q>1.5$ & 228.6 & 149.7 & 2.0 & 2.1 & 0.6 & 1.4 \\
\hline Quasar & Median & & & & & & \\
\hline$z($ all $)$ & QAll & 168.5 & 131.2 & 1.3 & 1.3 & 0.4 & 2.0 \\
\hline$z$ (all) & $Q \leq 1.5$ & 196.9 & 157.5 & 1.2 & 1.2 & 0.5 & 2.2 \\
\hline$z$ (all) & $Q>1.5$ & 153.8 & 93.7 & 1.8 & 1.9 & 0.3 & 1.9 \\
\hline$z \geq 0.3$ & QAll & 164.7 & 128.3 & 1.3 & 1.3 & 0.4 & 2.0 \\
\hline$z \geq 0.3$ & $Q \leq 1.5$ & 182.5 & 153.4 & 1.2 & 1.2 & 0.5 & 2.3 \\
\hline$z \geq 0.3$ & $Q>1.5$ & 130.2 & 93.7 & 1.8 & 1.9 & 0.4 & 1.9 \\
\hline
\end{tabular}

${ }^{*}$ For different redshift, $Q$ and $R$ subsamples for radio-loud quasars, and FR-II radio galaxies.

Note that for the comparison we used the ratio obtained from the median value data due to the wide dispersion of the $D$-values.

It should be noted that the original sample is quite heterogeneous, and contains both lobe- and core- dominated quasars (which may also exhibit different types of $D / R \& D / Q$ relations) and covers a wide range of redshifts $(0.0 \leq z \leq 2.87)$ and luminosities $\left(38.86 \leq \log P_{178 \mathrm{MHz}} \leq 48.89\right.$, a 7 orders of magnitude difference). This implies a wide range of plausible different host galaxies with differing environment). We believe that binning will smooth out randomly induced characteristics/values in the radio source parameters, and may reveal possible correlations between parameters of radio sources. One possible source of error is the binning range which was done to obtain equal representation in each bin (e.g see Ubachukwu 1998; Onuchukwu \& Ubachukwu 2013).

We divided each subsample into 10 (this choice is arbitrary, though motivated by the fact that the histogram plots were arranged into 10 bins). We evaluated the averages (mean and median) of each bin, which we used in the regression analysis shown in Table 6 . In the power-law fitted to the $D-Q / D-R$ relations, we have assumed that the power-law index is an indication of the strength of the relation, while the associated error indicates the level of scatter.

The $D-Q$ relation using the mean and the median values of the binned samples indicates a strong anti-correlation for both quasar and galaxy subclasses, except for the quasar subsample with 
TABLE 5

\begin{tabular}{|c|c|c|c|c|c|c|c|}
\hline & & $D_{\mathrm{G}} / D_{\mathrm{Q}}$ & $D_{\mathrm{G}} / D_{\mathrm{Q}}$ & $Q_{\mathrm{G}} / Q_{\mathrm{Q}}$ & $Q_{\mathrm{G}} / Q_{\mathrm{Q}}$ & $R_{\mathrm{G}} / R_{\mathrm{Q}}$ & $R_{\mathrm{G}} / R_{\mathrm{Q}}$ \\
\hline & & $R \leq 1.0$ & $R>1.0$ & $R \leq 1.0$ & $R>1.0$ & $R \leq 1.0$ & $R>1.0$ \\
\hline \multicolumn{8}{|l|}{ Median } \\
\hline$z($ all $)$ & $Q(\mathrm{All})$ & 1.5 & 2.1 & 1.0 & 0.9 & 1.5 & 0.7 \\
\hline$z($ all $)$ & $Q \leq 1.5$ & 1.4 & 2.0 & 1.0 & 1.0 & 1.6 & 0.6 \\
\hline$z($ all $)$ & $Q>1.5$ & 1.5 & 1.6 & 1.1 & 0.9 & 1.6 & 0.7 \\
\hline$z \geq 0.3$ & $Q($ All $)$ & 1.2 & 2.0 & 1.0 & 0.9 & 1.3 & 0.7 \\
\hline$z \geq 0.3$ & $Q \leq 1.5$ & 1.0 & 2.1 & 1.0 & 1.0 & 1.3 & 0.6 \\
\hline$z \geq 0.3$ & $Q>1.5$ & 1.8 & 1.6 & 1.1 & 1.1 & 1.5 & 0.7 \\
\hline \multicolumn{8}{|l|}{ Mean } \\
\hline$z($ all $)$ & $Q(\mathrm{All})$ & 1.7 & 1.8 & 0.9 & 0.8 & 1.3 & 0.5 \\
\hline$z($ all $)$ & $Q \leq 1.5$ & 1.4 & 1.5 & 1.0 & 1.0 & 1.4 & 0.4 \\
\hline$z($ all $)$ & $Q>1.5$ & 1.6 & 1.7 & 0.9 & 0.9 & 1.3 & 0.9 \\
\hline$z \geq 0.3$ & $Q($ All $)$ & 1.2 & 1.8 & 1.0 & 0.9 & 1.2 & 0.6 \\
\hline$z \geq 0.3$ & $Q \leq 1.5$ & 1.0 & 1.7 & 1.0 & 1.0 & 1.2 & 0.4 \\
\hline$z \geq 0.3$ & $Q>1.5$ & 1.6 & 1.4 & 1.0 & 0.9 & 1.1 & 0.8 \\
\hline
\end{tabular}

${ }^{*}$ For different redshift and $Q$ subsamples using the average (mean and median) values.

$Q \leq 1.5$ for both $z \geq 0.3$ and all $z$ bins, which suggest no correlation (with $r \approx-0.1 \ldots 0.0$ ). This result is consistent with beaming and orientation effects for both quasars and galaxies, even at large scales, and seems independent of redshift. The power-law index is similarly strong for both redshift bins. The observed absence of any significant correlation for quasar subsamples with $Q \leq 1.5$ is an indication that for such less asymmetric quasars there are other important factors that interfere with the beaming effect.

The $D-R$ correlation is mildly strong and inverse for quasars, decreasing with increasing $Q$, while it is mild and direct for galaxies, and seems not to vary with $Q$. The fairly strong inverse $D-R$ correlation for quasars supports the beaming hypotheses while the observed direct $D-R$ correlation for galaxies suggests other factors at play in the evolution of this class of radio sources.

\section{CONCLUSION}

We have compared the radio size $(D)$ and radio asymmetry parameters $Q / R$ relations based on the pure orientation and relativistic beaming unification scheme for FR-II radio galaxies and radio loud quasars. Using the median values, our results in general indicate that the projected linear sizes of the radio sources are smaller at higher redshift and at larger $Q$ values, with the linear size of quasars generally smaller than those of galaxies across all $Q$, $z$ and $R$ bins. Moreover, the linear sizes of galaxies seem smaller at lower values of $Q$ if $R \leq 1.0$ but become larger at higher values of $Q$ if $R>1.0$. For quasars, the linear size decreases with increasing $Q$, $R$ and $z$.

On the assumption that as $Q \longrightarrow Q_{\max }, R \longrightarrow$ $R_{\max }, D \longrightarrow D_{\text {min }}$ being a consequence of beaming and projection effects, our results suggest that beaming effect is more important in quasars than in galaxies. Moreover, the results of the $D-R$ regression analyses for the $Q$ and $z$ subsamples reveal that factors other than beaming and orientation are important in the interpretation of the evolution and dynamics of these radio sources. These other factors may include intrinsic asymmetries and environmentally induced asymmetries in radio sources (Ryś 1994, 2000; O'Dea 1998; Mackay's Rule-Mackay 1971; Ingham \& Morrison 1975; Gopal-Krishna \& Wiita 1996, 2000).

According to the orientation and unification scheme, the $Q / R$ analysis should indicate a stronger beaming effect in quasars than in galaxies. We note that in the sample we have used no effort was made originally to identify the "approaching" and "receding" side (Nilsoon 1988). The longer side was assumed to be the "approaching" side. Thus, a simple $Q / R$ analysis from this sample will give an inconclusive result. This is because, for most of the sources, to assume the longer side as the approaching side may be incorrect. Moreover, environmental factors (Onuchukwu 2017) that may shorten a side can 
TABLE 6

CORRELATION COEFFICIENT RESULTS FOR $D-Q / R$ RELATIONS.

\begin{tabular}{|c|c|c|c|c|c|}
\hline Mean & & $D / Q$ & $D / Q$ & $D / R$ & $D / R$ \\
\hline$z$ & $Q$ & $\mathrm{r}$ & & $\mathrm{r}$ & \\
\hline$z$ (All) & Gs(ALL $Q)$ & -0.8 & $D \propto Q^{-9.3 \pm 0.3}$ & 0.4 & $D \propto R^{1.7 \pm 0.5}$ \\
\hline$z(\mathrm{All})$ & $\operatorname{Qs}(\operatorname{ALL} Q)$ & -0.9 & $D \propto Q^{-8.6 \pm 0.2}$ & -0.9 & $D \propto R^{-2.4 \pm 0.2}$ \\
\hline$z(\mathrm{All})$ & $\operatorname{Gs}(Q \leq 1.5)$ & -0.2 & $D \propto Q^{-5.9 \pm 0.5}$ & 0.2 & $D \propto R^{0.8 \pm 0.5}$ \\
\hline$z(\mathrm{All})$ & $\operatorname{Qs}(Q \leq 1.5)$ & -0.1 & $D \propto Q^{-2.0 \pm 0.5}$ & -0.8 & $D \propto R^{-1.4 \pm 0.3}$ \\
\hline$z(\mathrm{All})$ & $\operatorname{Gs}(Q>1.5)$ & -0.7 & $D \propto Q^{-9.1 \pm 0.5}$ & 0.2 & $D \propto R^{0.5 \pm 0.7}$ \\
\hline$z(\mathrm{All})$ & $\operatorname{Qs}(Q>1.5)$ & -0.5 & $D \propto Q^{-4.6 \pm 0.4}$ & 0.2 & $D \propto R^{0.4 \pm 0.5}$ \\
\hline$z \geq 0.3$ & Gs(ALL $Q$ ) & -0.9 & $D \propto Q^{-6.9 \pm 0.3}$ & 0.5 & $D \propto R^{1.5 \pm 0.4}$ \\
\hline$z \geq 0.3$ & $\operatorname{Qs}(\operatorname{ALL} Q)$ & -0.7 & $D \propto Q^{-5.8 \pm 0.3}$ & -0.8 & $D \propto R^{-2.1 \pm 0.3}$ \\
\hline$z \geq 0.3$ & $\operatorname{Gs}(Q \leq 1.5)$ & -0.4 & $D \propto Q^{-8.6 \pm 0.4}$ & 0.2 & $D \propto R^{0.6 \pm 0.5}$ \\
\hline$z \geq 0.3$ & $\operatorname{Qs}(Q \leq 1.5)$ & 0.0 & $D \propto Q^{-0.5 \pm 0.4}$ & -0.8 & $D \propto R^{-1.2 \pm 0.3}$ \\
\hline$z \geq 0.3$ & $\operatorname{Gs}(Q>1.5)$ & -0.6 & $D \propto Q^{-3.6 \pm 0.4}$ & 0.4 & $D \propto R^{0.8 \pm 0.5}$ \\
\hline$z \geq 0.3$ & $\operatorname{Qs}(Q>1.5)$ & -0.3 & $D \propto Q^{-2.5 \pm 0.5}$ & 0.3 & $D \propto R^{0.9 \pm 0.5}$ \\
\hline Median & & $D / Q$ & $D / Q$ & $D / R$ & $D / R$ \\
\hline$z$ & $Q$ & $\mathrm{r}$ & & $\mathrm{r}$ & \\
\hline$z(\mathrm{All})$ & Gs(ALL $Q$ ) & -0.5 & $D \propto Q^{-9.5 \pm 0.5}$ & 0.4 & $D \propto R^{4.4 \pm 0.5}$ \\
\hline$z($ All $)$ & Qs $(\operatorname{ALL} Q)$ & -0.8 & $D \propto Q^{-10.8 \pm 0.3}$ & -0.6 & $D \propto R^{-2.2 \pm 0.4}$ \\
\hline$z(\mathrm{All})$ & $\operatorname{Gs}(Q \leq 1.5)$ & -0.4 & $D \propto Q^{-8.4 \pm 0.4}$ & 0.2 & $D \propto R^{1.8 \pm 0.5}$ \\
\hline$z(\mathrm{All})$ & $\operatorname{Qs}(Q \leq 1.5)$ & -0.1 & $D \propto Q^{-2.5 \pm 0.4}$ & -0.5 & $D \propto R^{-1.5 \pm 0.4}$ \\
\hline$z(\mathrm{All})$ & $\operatorname{Gs}(Q>1.5)$ & -0.6 & $D \propto Q^{-9.7 \pm 0.5}$ & 0.2 & $D \propto R^{0.7 \pm 0.7}$ \\
\hline$z(\mathrm{All})$ & $\operatorname{Qs}(Q>1.5)$ & -0.3 & $D \propto Q^{-3.8 \pm 0.4}$ & -0.2 & $D \propto R^{-0.4 \pm 0.5}$ \\
\hline$z \geq 0.3$ & Gs(ALL $Q$ ) & -0.7 & $D \propto Q^{-6.4 \pm 0.4}$ & 0.8 & $D \propto R^{3.7 \pm 0.3}$ \\
\hline$z \geq 0.3$ & $\operatorname{Qs}(\operatorname{ALL} Q)$ & -0.9 & $D \propto Q^{-10.5 \pm 0.2}$ & -0.5 & $D \propto R^{-1.9 \pm 0.4}$ \\
\hline$z \geq 0.3$ & $\operatorname{Gs}(Q \leq 1.5)$ & -0.5 & $D \propto Q^{-7.4 \pm 0.4}$ & 0.4 & $D \propto R^{1.5 \pm 0.4}$ \\
\hline$z \geq 0.3$ & $\operatorname{Qs}(Q \leq 1.5)$ & 0.0 & $D \propto Q^{0.6 \pm 0.4}$ & -0.2 & $D \propto R^{-0.5 \pm 0.4}$ \\
\hline$z \geq 0.3$ & $\operatorname{Gs}(Q>1.5)$ & -0.6 & $D \propto Q^{-3.6 \pm 0.4}$ & 0.4 & $D \propto R^{0.7 \pm 0.5}$ \\
\hline$z \geq 0.3$ & $\operatorname{Qs}(Q>1.5)$ & -0.4 & $D \propto Q^{-4.4 \pm 0.4}$ & -0.2 & $D \propto R^{-0.4 \pm 0.4}$ \\
\hline
\end{tabular}

${ }^{*}$ For different redshift and $Q$ subsamples for radio-loud quasars and FR-II radio galaxies.

also brighten the side, creating an anti-correlation in $Q / R$ relation, but a more positive correlation in the $D / R$ relation.

The authors acknowledge the ASN (Astronomical Society of Nigeria) for providing the platform for exchanging ideas with colleagues and the contribution of the anonymous referee to improve this work.

\section{REFERENCES}

Antonucci, R. 1993, ARA\&A, 31, 473

Arshakain, T. G. \& Longair, M. S. 2000, MNRAS, 311, 846

Barthel, P. D. 1989, ApJ, 336, 606

Best, P. N., Bailer, M. D., Longair, M. S., \& Riley, J. M. 1995, MNRAS, 275, 1171

Fanaroff, B. \& Riley, J. M. 1974, MNRAS, 167, 31P

Garrington, S. T. \& Conway, R. G. 1991, MNRAS, 250, 198
Gopal-Krishna \& Wiita, P. J. 1996, ApJ, 467, 191 2000, A\&A, 363, 507 2004, e-print arXiv:astro-ph/0409761

Ingham, W. \& Morrison, P. 1975, MNRAS, 173, 569

Jeyakumar, S. \& Saikia, D. J. 2000, MNRAS, 311, 397J

Jeyakumar, S., Wiita, P. J., Saikia, D. J., \& Hooda, J. S. 2005, A\&A, 432, 823J

Laing, R. A. 1988, Natur, 331, 149

Mackay, C. D. 1971, MNRAS, 154, 209

Macklin, J. T. 1981, MNRAS, 196, 967

McCarthy, P. J., van Breugel, W., \& Kapahi, V. K. 1991, ApJ, 371, 478

Nilsson, K. 1998, A\&AS, 132, 31

O'Dea, C. P. 1998, PASP, 110, 493

Onah, C. I., Ubachukwu, A. A., Odo, F. C., \& Onuchukwu, C. C. 2018, RMxAA, 54, 271

Onuchukwu, C. C. 2014, Cosmological Evolution In Extended Extragalactic Radio Sources, Advances in Science and Technology, 8, 23 
2017, Ap\&SS, 362, 100

Onuchukwu, C. C. \& Ubachukwu, A. A. 2013, Ap\&SS, 344, 211, DOI 10.1007/s10509-012-1325-x

Priya, A., Saikia, D. J., Singh, M., \& Chandola, H. C. 2012, MNRAS, 426, 758

Rees, M. J. 1967, MNRAS, 135, 345

Ryś, S. 1994, A\&A, 281, 15 2000, A\&A, 355, 79

Ryle, M. \& Longair, M. S. 1967, MNRAS, 136, 123

Safouris, V., Subrahmanyan, R., Bicknell, G. V., \& Saripalli, L. 2009, MNRAS, 393, 2

Saikia, D. J., Jeyakumar, S. K., Mantovani, F., et al. 2003, PASA, 20, 50

Scheuer, P. A. G. \& Readhead, A. C. S. 1979, Natur, 277,
182

Smith, E. P. \& Heckman, T. M. 1989, BAAS, 21Q, 777

Subrahmanyan, R., Saripalli, L., Safouris, V., \& Hunstead, R. W. 2008, ApJ, 677, 63

Ubachukwu, A. A. 1996, Ap\&SS, 236, 167 1998, Ap\&SS, 254, 23 2002, Ap\&SS, 279, 251

Ubachukwu, A. A. \& Ogwo, J. N. 1998, AJP, 51, 143

Urry, C. M. \& Padovani, P. 1995, PASP, 107, 803

Valtonen, M. J. 1979, ApJ, 231, 312

Wardle, J. F. C. \& Aaron S. E. 1997,MNRAS286, 425

Willot, C. J., Rawlings, S., Blundell, K. M., \& Lacy, M. 1998, MNRAS, 300, 625

Zensus, J. A. 1997, ARA\&A, 35, 607

Onuchukwu, C. C.: Department Of Industrial Physics, Chukwuemeka Odumegwu Ojukwu University, Anambra State, Nigeria (onuchukwu71chika@yahoo.com).

Ubachukwu, A. A.: Department of Physics and Astronomy, Faculty of Physical Sciences, Carver Building, University of Nigeria Nsukka, Enugu State, Nigeria. 